\title{
Intersections
}

Canadian Journal of Music

Revue canadienne de musique

\section{Linda Cummins. 2006. Debussy and the Fragment. Amsterdam : Rodopi. 192 p. ISBN 9789042020658 (pbk.), 9042020652 (pbk.) (couverture souple)}

\section{François de Médicis}

Volume 29, numéro 2, 2009

URI : https://id.erudit.org/iderudit/1000043ar

DOI : https://doi.org/10.7202/1000043ar

Aller au sommaire du numéro

Éditeur(s)

Canadian University Music Society / Société de musique des universités canadiennes

ISSN

1911-0146 (imprimé)

1918-512X (numérique)

Découvrir la revue

Citer cet article

de Médicis, F. (2009). Linda Cummins. 2006. Debussy and the Fragment. Amsterdam : Rodopi. 192 p. ISBN 9789042020658 (pbk.), 9042020652 (pbk.)

(couverture souple). Intersections, 29(2), 115-140.

https://doi.org/10.7202/1000043ar

All Rights Reserved (C Canadian University Music Society / Société de musique des universités canadiennes, 2010
Ce document est protégé par la loi sur le droit d'auteur. L'utilisation des services d'Érudit (y compris la reproduction) est assujettie à sa politique d'utilisation que vous pouvez consulter en ligne.

https://apropos.erudit.org/fr/usagers/politique-dutilisation/ 


\section{ESSAI CRITIQUE / REVIEW ESSAY}

Linda Cummins. 2006. Debussy and the Fragment. Amsterdam : Rodopi. 192 p. ISBN 9789042020658 (pbk.), 9042020652 (pbk.) (couverture souple).

Parmi les récentes études publiées sur Debussy, l'ouvrage que Linda Cummins consacre au fragment dans l'œuvre du compositeur français s'avère une des contributions les plus rafraîchissantes, autant par la valeur de ses analyses et de ses rapprochements entre la musique et d'autres arts que par le pouvoir suggestif de la perspective qu'elle ouvre au lecteur. Le fragment a fait l'objet d'importants travaux musicologiques, proposant d'intéressants parallèles entre musique et littérature, mais aucune étude approfondie ne s'était encore intéressée au fragment chez Debussy. Le fragment littéraire a d'abord été théorisé par les écrivains de l'école d'Iéna, en particulier Friedrich Schlegel (1772-1829), qui a lui-même écrit des séries de fragments. Dans ces recueils, comme les 127 fragments qu'il a publiés en 1897 , de courts aphorismes et des textes plus longs s'enchaînent et forment un système dynamique qui rejette la conception aristotélicienne de l'art (cohérence, unité, complétude, logique des enchaînements). Ils valorisent plutôt la discontinuité, la contradiction, et développent une nouvelle logique résultant du choc de parties autonomes, du mélange des genres (en littérature : le dialogue, la réflexion philosophique, le lyrisme poétique), de même qu'ils cultivent l'inachèvement, l'ouverture, la suggestivité, la polysémie et le dynamisme du sens. Cummins cite à propos le fragment 116 de Schlegel : "La poésie romantique est encore à l'état de devenir ; c'est là, en fait, sa véritable essence : qu'elle demeure toujours en devenir, et jamais complétée $^{1} »$ (p. 11). Des travaux de Daverio, Rosen, Kallberg, Hoeckner, Satyendra et Ferris ayant déjà abordé le fragment musical à l'époque romantique, il était logique de poursuivre la recherche chez Debussy, subtil héritier de Schumann et Chopin ${ }^{2}$. Mais l'ouvrage de Linda Cummins met aussi en rapport le fragment musical debussyste avec les développements de l'étude du fragment en études littéraires.

Les réflexions que m’ont suggéré la lecture du livre m’ont naturellement amené à dépasser le cadre du strict compte rendu et d'embrasser le genre de la recension sous la forme d'essai (le Review Essay en anglais), en raison de la structure du livre et de la nature de son sujet. Déjà, les chapitres du livre se présentent comme une série de fragments. Il mapparaissait trop restrictif de simplement en rapporter les idées, les ruminations qu'elles m'inspiraient

1 "The Romantic kind of poetry is still in the state of becoming; that, in fact, is its real essence: that it should forever be becoming and never be completed ».

2 Voir Daverio 1993, Rosen 1995, Kallberg 1996, Hoeckner 1997, Satyendra 1997 et Ferris 2000. 
suggéraient des commentaires débordant le cadre de la matière présentée. Cet article est divisé en deux parties : la première résume le contenu du livre sans en approfondir la matière; la seconde reprend trois analyses de Linda Cummins et les développe dans des directions suggérées par l'esprit du fragment ou selon des avenues indiquées par l'auteure. Après tout, le genre de l'essai lui-même s'inscrit dans la tradition du fragment, avec son cours fluide qui entremêle spéculations et digressions, comme le rapporte d'ailleurs Cummins en lien avec un de ses représentants les plus illustres, Michel de Montaigne. Je précise que les réflexions qui suivent ne se veulent pas une critique, mais témoignent au contraire de la grande stimulation que j’ai éprouvée à la lecture du livre de Cummins (dans l'esprit même du fragment, dont l'impact réside autant dans la contribution spécifique de son énoncé que dans sa force d'évocation).

Linda Cummins est professeure à la University of Alabama à Tuscaloosa et elle se spécialise à la fois dans l'œuvre de Debussy et dans la musique médiévale. Son livre Debussy and the Fragment, dérivé de la thèse qu'elle a complétée en 2001 à la Louisiana State University (sous la direction d'un éminent debussyste, Matthew Brown), est composé de six chapitres : le premier fournit une perspective historique sur l'utilisation du fragment en littérature et en musique ; et les cinq suivants abordent différentes techniques ou concepts associés au fragment dans la musique de Debussy : les débuts et fins d'un morceau hors de la tonique, ou sur une harmonie tonalement ambiguë ; les œuvres à caractère grec ou arcadien (où la notion bien connue dans les études debussystes de l'arabesque est abordée à partir d'une perspective mallarméenne) ; l'esquisse, comme titre ou sous-titre d'œuvres de Debussy, mais aussi comme genre musical ; l'autocitation, comme cas particulier de la citation d'un matériau exogène, étranger au tissu d'une œuvre; et le genre du prélude, dans le rapport qui lie les deux recueils de Debussy à la fois à Chopin, à la tradition de l'art de préluder, et à l'écriture de groupes de pièces.

Longtemps, le fragment littéraire a été associé principalement à Friedrich Schlegel et à la littérature de l'Allemagne romantique. Comme le fait valoir Linda Cummins, les travaux musicologiques consacrés jusqu'à maintenant au fragment en musique sont surtout circonscrits à la même période, comme en témoignent les travaux portant sur Chopin (Rosen, Kallberg), Schumann (Daverio, Rosen, Ferris) ou Liszt (Satyendra), avec néanmoins une ouverture vers Debussy3. Howat a situé Debussy dans la filiation de Chopin, Rosen dans celle de Schumann; et dans une lettre à son éditeur, le compositeur français place lui-même son livre d'Images dans le sillage des œuvres pour piano de Chopin et Schumann ${ }^{4}$. Le chapitre 1 rend compte d'études récentes (où une place spéciale revient à l'important ouvrage édité par Lawrence D. Kritzman et Jeanine Parisier Plottel) qui tracent un panorama beaucoup plus vaste des manifestations du fragment littéraire, s'étendant du $\mathrm{XIV}^{\mathrm{e}}$ au XX $\mathrm{XX}^{\mathrm{e}}$ siècles, et dont les principaux

3 Les ouvrages de Rosen, Kallberg, Daverio, Ferris et Satyendra, sont cités plus haut, à la note 2.

4 Howat 1992, Rosen 1995 ; lettre du 11 septembre 1905 de Debussy à Jacques Durand (Debussy 2005, 919). 
jalons retenus par Cummins sont les Canzoniere de Pétrarque (1304-1374), les Essais de Montaigne (1533-1592), Gargantua de Rabelais (1494-1553), Don Quichotte de Cervantes (1547-1616), Jacques le fataliste de Diderot (1713-1784), The Life and Opinions of Tristram Shandy de Sterne (1713-1768), les Fragments de l'Athenaeum et Lucinde de Schlegel (1772-1829), et des auteurs français du XIX ${ }^{\mathrm{e}}$ siècle (comme Lautréamont ou Nerval) (voir Kristman et Plottel, 1981). À la fin du XIX ${ }^{e}$ siècle, les écrivains symbolistes, en bons héritiers des romantiques, poursuivent cette tradition. Si la littérature occupe une grande place dans la discussion, Cummins n'omet pas de citer les arts visuels (par exemple, le nom d'Odilon Redon affleure à plusieurs reprises dans le livre).

Dans les chapitres 2 à 6 , le livre se concentre sur Debussy, mais le chapitre 1 leur sert de toile de fond, justifiant les allers-retours entre l'écriture musicale du compositeur français et la tradition littéraire du fragment. Au cours de ces pages, l'auteure fait preuve d'une belle sensibilité littéraire, puisant dans sa vaste connaissance d'écrits sur la littérature, et proposant des analyses de textes éclairantes. Elle aborde une série de techniques qui touchent d'une manière ou d'une autre au fragment : les commencements in media res et les conclusions hors tonique concernent principalement le début et la fin des fragments, alors que l'arabesque et l'esquisse touchent davantage à l'organisation du centre de l'œuvre. Dans les Préludes pour piano, Cummins observe l'influence du paratexte sur la réception du fragment. L'auteure discute en détail les techniques du début in media res et des finales hors de la tonique avec des passages de deux Ariettes oubliées de Debussy, les mélodies « Green » et « Spleen », et du prélude pour piano Canope. Je vais revenir sur ces œuvres plus loin dans la partie essai de cet article. La pratique du début in media res chez le compositeur français est située dans le contexte d'œuvres romantiques telles que le Prélude en la mineur, op. $28 \mathrm{n}^{\circ} 2$ de Chopin, l'Intermezzo en si bémol mineur, op. $118 \mathrm{n}^{\circ} 1 \mathrm{de}$ Brahms. Pour l'obscurcissement tonal de la conclusion d'une œuvre, Cummins mentionne le Prélude n 3 de Bach, BWV 999, le Prélude en fa majeur, op. 28 et la Mazurka, op. $30 \mathrm{n}^{\circ} 2$ de Chopin, et la mélodie de Liszt, « S'il est un charmant gazon ». Dans le premier lied du cycle de Schumann Dichterliebe op. 48, «Im wunderschönen Monat Mai », la tonique est évitée au début comme à la fin.

Le chapitre 3 porte sur l'Arcadie et l'arabesque. Cummins s'intéresse à l'inspiration de la Grèce antique sur l'écriture du fragment des poètes français de la fin du XIX ${ }^{\mathrm{e}}$ siècle et de Debussy. Elle observe que la Grèce antique a considérablement inspiré les parnassiens, décadents et symbolistes, et qu'elle leur fournit le thème de nombreux fragments littéraires. Cette Grèce en partie imaginée offre à ces littérateurs un idéal de retenue, de pudeur, de maîtrise des passions ; paradoxalement, ce monde pré-chrétien est aussi associé à l'épanouissement de la liberté individuelle et à l'expression d'une sexualité débridée, dénuée d'inhibition (comme avec la Bilitis de Pierre Louÿs). L’Arcadie fournit en outre un répertoire abondant de figures mythologiques, qui s'étaient chargées de multiples attributs et avaient accumulé un sens pluriel au cours de nombreux siècles de réception et de réappropriation. Cummins s'intéresse plus précisément à deux auteurs inspirés par ces thèmes arcadiens, Stéphane Mallarmé et Pierre Louÿs, et à deux œuvres de Debussy qu'ils ont inspirées, le 
Prélude à l'après-midi d'un faune et le "Tombeau des Naïades" (la troisième des Chansons de Bilitis). Dans les faunes de Mallarmé et Debussy, Cummins aborde également l'écriture en arabesque, un important concept mallarméen. L'arabesque possède deux sens, celui d'un ornement et celui d'un principe de continuité sous-tendant la création. Chez Debussy et Mallarmé, l'œuvre décrit une arabesque par sa ligne narrative sinueuse, poursuivant un cours fait d'interruptions, de digressions et de déformations imposées à des conventions sous-jacentes. Pour le poète, chaque art est doté d'une spécificité propre et suit sa propre arabesque. Mais replacé dans la perspective de l'art total, il constitue l'un des rameaux d'une grande arabesque totalisante. On peut donc concevoir l'apport musical de Debussy comme une arabesque qui se superpose et s'entrelace à celle de Mallarmé.

Autre manifestation de l'inspiration arcadienne, le livre des Chansons de Bilitis de Pierre Louÿs s'inscrit dans une tradition populaire chez de nombreux auteurs de fragments (comme Bacon, Hamann, Diderot, ou Laurence Sterne) : celui du canular littéraire, où une œuvre originale se présente comme la traduction de fragments récemment redécouverts d'un livre ancien. Louÿs, fidèle à ce genre, présente un document fictif troué de lacunes. Au début in media res du texte du « Tombeau des Naïades » de Louÿs, Debussy répond par le procédé du début hors tonique.

Le chapitre 4 s'intéresse à deux ouvres qui portent le titre ou le sous-titre d'esquisse, La Mer (esquisses symphoniques) et D'un cahier d'esquisse. Cummins prend ce terme dans le sens d'un emprunt à l'art pictural, et montre que ces œuvres adoptent un style impromptu, inachevé, similaire à celui qu'on retrouve dans les esquisses visuelles. L'auteure rapporte sous toute réserve le témoignage de Ricardo Viñes à qui Debussy aurait affirmé vouloir écrire des œuvres d'une telle liberté qu'elles donnent l'impression d'être improvisées. Je ferais remarquer qu'une œuvre antérieure, exécutée à Paris quelques années auparavant, porte déjà le titre de $\mathrm{La} \mathrm{Mer}$, et le même sous-titre "d'esquisse symphonique ». Il s'agit d'un poème symphonique du compositeur belge Paul Gilson. Évidemment, cela ne signifie pas que Gilson proposait un sens différent de celui de Debussy, ou que le compositeur français donnait à son sous-titre un sens ignoré de son prédécesseur. Cummins remarque que dans les beaux-arts, un dessin tiré d'un carnet d'esquisse reprend souvent un thème exploré dans d'autres croquis du même recueil. Elle rapproche cette pratique d'une découverte de Roy Howat, qui a démontré que D’un cahier d'esquisse devait initialement faire partie d'un cycle de trois pièces (conjointement avec l'Isle joyeuse et Masques) 5 . Et effectivement, certains motifs circulent d'une œuvre aux autres.

Le chapitre 5 observe l'impact esthétique d'un procédé fréquent chez Debussy, celui de l'autocitation. Cummins n'y voit pas un simple trait stylistique, ni un tic de langage, mais s'intéresse à l'effet de rupture qu'il produit au sein d'une œuvre individuelle d'une part, et à l'effet de réseautage qui en résulte entre deux ou plusieurs œuvres distinctes d'autre part. Elle compare ce procédé à

5 Howat 1995 ; l'extrait en question est cité en p. 124, note 18. 
celui d'écrivains qui font figurer un même personnage dans plusieurs romans ou récits ${ }^{6}$.

Cummins rapproche ce trait debussyste du fameux «Florestan » du Carnaval op. 9 de Schumann, qui cite la valse des Papillons op. 2. Daverio (1993) et Rosen (1995) s'étaient déjà intéressés à l'effet de rupture que cela produit dans l'œuvre du compositeur allemand. Pour Rosen, dont Cummins rapporte l'interprétation, l'insertion du fragment de Papillon est originale non seulement parce qu'il s'agit d'une citation, mais aussi parce que Schumann fait ressortir le contraste du matériau étranger dans la texture de la nouvelle œuvre (un peu comme s'il le mettait entre guillemets) : il le fait donc sonner comme une citation. Et Cummins ajoute sa propre interprétation : "Papillons fragmente 'Florestan', mais en plus, il relie non seulement deux pièces individuelles de Schumann, mais également deux recueils de miniatures : Papillons et Carnaval7 » (p. 139). Cummins discute deux exemples de citation dans la musique de Debussy : un passage de la scène 3 de l'acte II de Pelléas et Mélisande, repris dans « Nuages" (le premier des Nocturnes pour orchestre) ; et celui de « Le Matin d'un jour de fête " (dernier mouvement d'Ibéria), inséré dans la "Sérénade interrompue» (du premier livre des Préludes pour piano). Dans le premier exemple, elle développe davantage le réseau de réminiscences pour inclure des œuvres d'autres compositeurs, antérieures et postérieures à celles de Debussy : la troisième mélodie de Sans soleil (Без солнца) de Moussorgski, et l'ouverture du Rossignol (Соловей) de Stravinski. Elle décrit deux effets associés à la citation : celui d'une signature apposée par le créateur à même son œuvre, et celui d'une intrusion autorielle, qui crève l'illusion de la fiction ${ }^{8}$.

En définitive, cette question complexe demeure difficile à cerner, comme le reconnaît Cummins : "L'emprunt, la citation et même l'autocitation soulèvent souvent des questions complexes de chronologie, d'influence et de simple

6 Cummins écrit : "A web that is literary in purpose and character, creating musical ideas that appear in multiple compositions, much as some nineteenth-century writers created characters who appeared as either major or minor figures in multiple stories. Each work is complete within itself and yet not complete since each quotation, each reappearing character, directs the attention of the reader outside the work at hand. The effect is one of fragmentation at the level of the individual work, but also of connection, binding multiple works in a larger context » (p. 139). ("Un réseau qui se propose un but et une inspiration littéraire, créant des idées musicales reprises dans plusieurs œuvres, tout comme les écrivains du XIX ${ }^{\mathrm{e}}$ siècle créaient des personnages réapparaissant comme personnages principaux ou mineurs dans de nombreux récits. Chaque œuvre est complète en ellemême sans être cependant complète puisque chaque citation, chaque personnage récurrent, dirige l'attention du lecteur au-delà de l'œuvre immédiate. Il en résulte un effet de fragmentation au niveau de l'œuvre individuelle, mais aussi de connexion, liant différentes œuvres dans un contexte plus vaste ».)

7 «Papillons fragments 'Florestan', but it also connects-not only two of Schumann's individual works, but two of his collections of minatures : Papillons and Carnaval ».

8 Cummins cite également des exemples d'autocitation en peinture : le miroir inséré dans Les Époux Arnolfini de Jan van Eyck; les tableaux accrochés aux murs dans Le grand intérieur rouge d'Henri Matisse et dans le Portrait d'Émile Zola d'Édouard Manet (où l'on distingue à l'arrière-plan l'Olympia). 
coïncidence. Toutes ces connexions, ces bribes de souvenirs rendus publics appellent des recherches plus approfondies 9 » (p. 150).

Le dernier chapitre aborde le rôle du paratexte dans les collections de fragments que constituent les deux livres de Préludes pour piano. Pour Gérard Genette (1987), le paratexte renvoie à toute une série d'annotations, de productions qui accompagnent un texte : le nom de l'auteur, le titre, la préface, les illustrations, etc. Cummins s'intéresse en particulier à deux éléments du paratexte : le titre des deux recueils, «Préludes », pour les attentes qu'il suscite auprès de l'auditeur; et les titres des préludes individuels, pour la signification qui résulte de leur disposition inhabituelle, à la fin de chaque pièce, entre parenthèses et suivant des points de suspension. En assemblant des pièces par groupes de douze et en leur conférant le titre de «Préludes», Debussy s'inscrit dans une prestigieuse tradition qu'illustrent plus particulièrement les préludes et fugues de Das Wohltemperierte Klavier de Bach et les Préludes, op. 28 de Chopin. À l'origine, le genre du prélude est associé à un caractère inachevé, car il a pour fonction d'introduire une autre pièce. Mais les Préludes de Chopin ont surpris les attentes de ses premiers auditeurs par le radicalisme de son style fragmentaire, causé par l'excessive brièveté de certaines pièces, et aux débuts ou fins tonalement ambigus d'autres d'entre elles. L'écriture fragmentaire des Préludes de Debussy s'inscrit dans la filiation de Chopin, mais elle est hybridée avec la tradition des pièces de caractère, par la longueur des pièces et par l'ajout de titres poétiques (comme les pièces des cahiers d'Estampes et d'Images du même compositeur). Cummins aborde également la question du plan tonal et de l'unification cyclique de l'ensemble des pièces. Contrairement à ce qu'on observe dans les groupes de préludes de Bach et de Chopin, les tonalités de ceux de Debussy ne suivent pas un ordre régulier exploitant systématiquement l'ensemble des tonalités majeures et mineures de la gamme chromatique. Et l'auteure met en garde contre les abus d'une perspective organiciste toujours très en faveur dans la musicologie actuelle : la présence de connexions, d'unification motivique ne doit pas faire sous-estimer la tension dynamique qu'instaure le fragment au sein du groupe de pièces, tirant les pièces individuelles à la fois dans un mouvement d'éclatement, de repli sur soi, et d'ouverture et d'interrelation avec les autres pièces du recueil, sans jamais atteindre l'unification complète.

Cummins discute l'origine des titres des Préludes de Debussy à partir d'une synthèse des travaux publiés sur le sujet. Si on excepte quatre titres dont l'origine reste inconnue, elle range les Préludes dans trois catégories principales : les pièces peuvent être nommées d'après des citations de poèmes déjà mis en musique par Debussy, d'après des objets personnels, ou d'après des souvenirs du compositeur. Par exemple, le Prélude «Les Sons et les parfums tournent dans l'air du soir » est un alexandrin emprunté au poème "Harmonie du soir » de Baudelaire (mis en musique dans les Cinq poèmes de Baudelaire) ; "Canope »

9 «Borrowing, quotation, even auto-quotation often raise complicated issues of timing, influence, and sheer coincidence. All these connections, these 'memories made public' in bits and pieces, invite further investigation ». 
renvoie aux urnes funéraires égyptiennes qui ornaient le manteau de la cheminée du compositeur; et «Feu d'artifice » évoque le souvenir que Debussy a conservé des fêtes du 14 juillet 1912. Il s'agit à chaque fois d'éléments familiers de la vie de Debussy, qui trahissent son goût de l'acquisition, de la collection, confirmé par les objets qui meublaient son intérieur, et reflété à la fois dans sa musique et par les propos de sa correspondance. Pour Cummins, les Préludes constituent donc " une représentation musicale de ce scrapbook du quotidien $^{10} »$ (p. 16o). L'auteure compare le Debussy casanier de la maturité avec l'esthète collectionneur et reclus, Des Esseintes, héros décadent du roman $A$ rebours de Joris-Karl Huysmans.

Pour expliquer l'emplacement du titre des préludes à la fin de chaque pièce, Cummins rapporte les hypothèses de différents auteurs et offre elle-même plusieurs interprétations. Il n'est pas nécessaire de toutes les rapporter ici, et je me contenterai de citer la dernière, qui termine très poétiquement le livre. Cummins propose de considérer ces titres comme un exemple de ce que Genette appelle des épigraphes terminales. Si ces épigraphes étaient placées en tête de l'œuvre comme c'est l'usage, elles vendraient trop tôt la mèche. Cet argument illustre un peu la fameuse maxime de Stéphane Mallarmé, "nommer un objet, c'est supprimer les trois quarts de la jouissance du poème qui est faite de deviner peu à peu : le suggérer, voilà le rêve... » (cité par Cummins, p. 165-166). Les Préludes se présentent donc comme des traces préservant des moments du monde intime et familier de Debussy, mais de manière allusive et parcellaire, ne subsistant qu'à l'état de vestiges fragmentaires.

Un bref résumé du contenu du livre de Cummins ne peut rendre justice aux analyses, qui abordent avec finesse et sensibilité la transposition du fragment en musique. La section essai qui suit va permettre de résumer l'argument de quelques analyses de l'auteure, et je m'autorise de leur suggestivité pour alimenter la réflexion qu'elles suscitent de quelques observations personnelles.

\section{'GREen' et 'SPLEEN'}

Dans son chapitre 2, Cummins discute le début hors tonique de deux Ariettes oubliées de Debussy pour illustrer la problématique du début in media res. Dans les Romances sans paroles de Verlaine, non seulement les deux poèmes " Green » et "Spleen » se présentent successivement, mais ils sont également couplés (les deux titres, avec leur assonance et leur emprunt similaire à la langue anglaise, l'annoncent déjà). Cummins montre que les deux mélodies que Debussy a composées sur ces textes sont aussi conçues comme une paire sur le plan musical. Lauteure considère les deux textes comme des illustrations représentatives du genre du fragment, et montre comment la forme musicale répond à l'écriture des deux poèmes, avec leurs effets de démembrement et de déchiquetage. Sur le plan littéraire, Cummins décrit le premier vers de « Green » comme l'énumération de parties de végétation déchirées, auquel est assimilé le

10 " Musical representations of this scrapbook of the every day ». 
cœur du narrateur dans les deux vers suivants : «Voici des fruits, des fleurs, des feuilles et des branches/Et puis voici mon cœur qui ne bat que pour vous./Ne le déchirez pas avec vos deux mains blanches [...]». Dans « Spleen», l'auteure observe que les strophes se composent non des quatrains ou tercets habituels, mais de courts distiques, comme si les quatrains avaient été coupés en deux. De plus, l'enchaînement des premiers distiques produit une discontinuité sur le plan du sens, qui suit des correspondances croisées entre les distiques 1 et 3 , et 2 et 4 (avec l'alternance entre des descriptions énumératives de la nature à l'imparfait de l'indicatif d'une part, et l'expression des états d'âme du narrateur induits par le comportement de sa bien-aimée au présent de l'indicatif d'autre part).

[Distique 1] Les roses étaient toutes rouges, Et les lierres étaient tout noirs.

[Distique 2] Chère, pour peu que tu te bouges, Renaissent tous mes désespoirs.

[Distique 3] Le ciel était trop bleu, trop tendre, La mer trop verte et l'air trop doux;

[Distique 4] Je crains toujours, ce qu'est d'attendre, Quelque fuite atroce de vous!

[Distique 5] Du houx à la feuille vernie, Et du luisant buis je suis las,

[Distique 6] Et de la campagne infinie, Et de tout, fors de vous. Hélas!

Cummins rend compte de l'effet fragmentaire de la réalisation musicale par le début hors tonique, mais je vais intégrer ces considérations avec d'autres éléments musicaux qu'elle omet pour soulever d'autres aspects de l'esthétique fragmentaire et essayer d'approfondir certains traits propres à l'écriture debussyste. D'abord, je suggérerais que «Spleen » se rattache à une importante tradition vocale qui peut aider à expliquer l'effet de début in media res: celle du récitatif et air (voir l'exemple 1). Le prélude du piano introduit les principaux motifs de l'œuvre (les segments " $\mathrm{x}$ » et " $\mathrm{y}$ »), dans un caractère improvisé et hésitant, caractéristique de l'accompagnement de récitatif, avec une finale laissée en suspens (à la mes. 4). Puis la voix fait son entrée avec un grand dépouillement (ponctué de quelques brèves interjections au piano dans le registre médian, sans basse) en déclamation recto tono. C'est seulement aux environs de la mes. 9 que la pièce semble débuter pour de bon, lorsque la main droite du piano introduit un patron d'accompagnement syncopé se superposant au motif « $\mathrm{x}$ », et que la voix émet une longue plainte descendante (qui se range dans une famille de gestes debussystes caractéristiques, que Jankélévitch qualifie de géotropiques ${ }^{11}$.

11 Voir les deux ouvrages de Vladimir Jankélévitch, Debussy et le Mystère (1949, 89-ff.) ; et Debussy et le Mystère de l'instant (1976, 44-ff). 

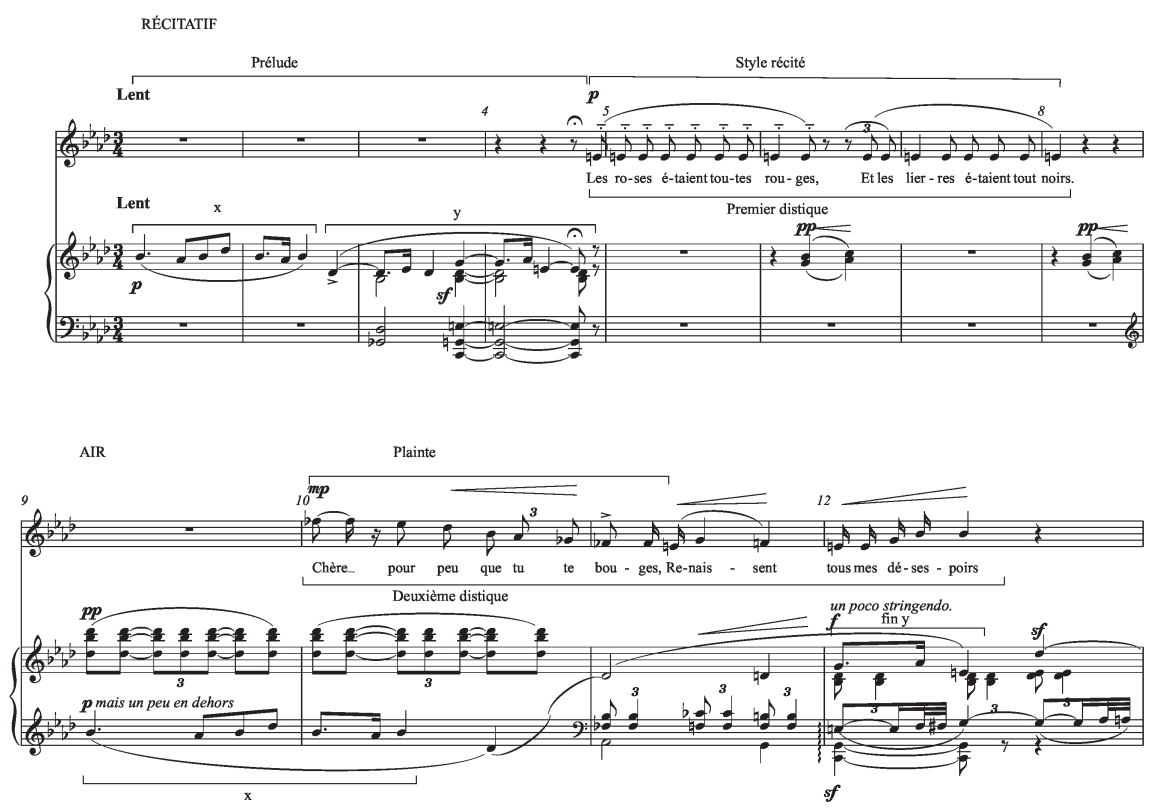

Exemple 1: Debussy, «Spleen », mes. 1-12.

Cette mélodie n'est pas la seule de Debussy à débuter dans le style " récitatif et air ", on peut également citer à titre d'exemples Souhait (1881), les airs de L'Enfant prodigue (1884), tels ceux de Lia et d'Azaël (ce qui est plus prévisible vue la proximité du genre de la cantate de Rome avec l'opéra), ou à une époque légèrement postérieure, "Recueillement » (1889). Ce procédé contribue à créer l'impression d'un début in media res, mais selon un climat particulier. En effet, je voudrais souligner que plus d'un moyen littéraire ou musical peuvent créer l'effet de débuter au cœur de l'action, et ceux-ci peuvent à leur tour susciter des effets esthétiques très variés. Dans certains récits ou actions dramatiques, la brusquerie du début projette le lecteur brutalement au cœur de l'intrigue, avant de lui fournir a posteriori les repères nécessaires pour se situer dans l'histoire. Cela a pour effet d'accuser la temporalité du récit et de produire un effet de mouvement emporté et de confusion plus ou moins marqué. Cela peut se retrouver même dans des œuvres aussi peu associées à l'esthétique du fragment que les opéras de Mozart. C'est le cas du début de Don Giovanni : au lever du rideau, Leporello fait le guet, et il a à peine le temps d'ébaucher une ariette qu'il est coupé par l'irruption de Don Giovanni, poursuivi par Donna Anna, que le seigneur avait tenté de séduire ; ensuite, le Commandeur s'interpose, provoque le séducteur en duel, et meurt, frappé par l'épée de son jeune adversaire ${ }^{12}$. À première vue, l'effet esthétique du début in media res dans "Spleen " est tellement différent que les deux ouvres semblent n'avoir rien en commun.

12 Mozart transpose musicalement l'effet littéraire à l'aide d'une Introduzione (l'équivalent d'un finale d'acte, mais placé au début de l'œuvre). Die Zauberflöte, qui propose comme Don Giovanni un début in media res, commence également par une Introduzione. 
Chez Debussy, la voix énonce le premier distique d'une récitation discrète avec un léger accompagnement, comme si la musique aidait l'auditeur à s'insinuer dans le cours du récit sans en avoir l'air. Cet exemple illustre la complexité des questions qui touchent aux rapports entre les techniques du fragment, le début in media res, l'esthétique et le style. C'est important d'en tenir compte non seulement pour différencier les styles, mais aussi pour expliquer l'apparente disparité de démarche et d'esthétique qu'on peut retrouver chez différents créateurs pratiquant l'écriture par fragment.

Si Cummins attribue le caractère fragmentaire du poème de Verlaine principalement à l'opposition des distiques, elle n'établit aucune corrélation dans la réalisation musicale de Debussy sur ce plan. Un parallèle est néanmoins discernable si on ne cherche pas à établir une correspondance trop étroite et systématique entre les moyens musicaux et l'opposition de sens dans le texte. En effet, la division des deux premiers distiques au moins est reflétée dans l'opposition vocale entre le style récité et la plainte (mes. 5-8 et 10-12). Cela met en lumière la fêlure fondamentale qui affecte la psyché du narrateur : d'une part, celui-ci tente de maîtriser son trouble, et d'autre part, on le voit submergé par une vague d'exaspération irrépressible. La réalisation musicale produit une caractérisation d'une grande justesse : la récitation recto tono donne l'impression du détachement (naturel ou forcé ?), bien adapté au caractère objectif du premier distique, quand le poète se contente de narrer ses observations. Et la plainte subite (dont le caractère soudain est souligné par son irruption dans le registre aigu au milieu du calme motif « $\mathrm{x}$ » de la mes. 9) souligne l'interpellation directe et le tutoiement de la bien-aimée ("Chère, [...] tu »), ainsi que l'aveu de faiblesse du deuxième distique. La partition cesse ensuite de souligner l'opposition des distiques, pour mettre en valeur d'autres aspects du texte, en particulier l'anxiété croissante du narrateur (rendue par un grand accroissement de tension musicale).

La fêlure du narrateur, que j'invoque pour rendre compte de l'effet littéraire de contraste des distiques, est également transposée musicalement à plus courte échelle encore, dans le caractère opposé des deux motifs du prélude de piano (toujours illustré sur l'exemple 1). Aux mes. 1-4, le segment « X » propose une ligne monodique, diatonique, unie, calme et lyrique, et le segment « $y$ » lui oppose un caractère trouble, avec sa ligne accompagnée, son rythme plus pointé, ses syncopes, son sforzando, et le chromatisme de son enchaînement d'accords à distance de triton. Les deux motifs vont être développés dans l'ensemble de la mélodie (le second est associé à de grandes accumulations de tension) : voir par exemple le retour des motifs « $\mathrm{x}$ » et $" \mathrm{y} »$ aux mes. 9 et suivantes ${ }^{13}$. L'association de « $y$ » avec le caractère tourmenté se manifeste de plusieurs manières : la fin de «y », qui est retardée jusqu'à la mes. 12, accompagne les paroles " [renaissent] tous mes désespoirs " (la résurgence du désespoir coïncide avec le retour

13 En ce qui concerne le motif " $\mathrm{X}$ ", on observe comment les deux éléments associés à la tentative de maîtrise de soi, initialement successifs, sont superposés au début de la $2^{\mathrm{e}}$ partie (mes. 18-19) : la récitation recto tono de la voix sur les paroles «Je crains toujours ce qu'est d'attendre " se combine au motif « $\mathrm{x}$ » (avec, comme au début, une suspension du registre grave et un retour subit à la nuance piano). 
dans le registre grave de la ligne de basse, qui avait été suspendue entre les mes. $5-10)$. Le trait ascendant de la voix à la mes. 12 renverse subitement la direction du mouvement, et l'accélération subite du rythme de doubles-croches illustre de façon suggestive la vague de désespoir qui assaille subitement le narrateur. Les mesures suivantes, non représentées dans l'exemple 1, développent le matériau «y » de la mes. 12. L'opposition entre les deux motifs semble évoquer la tension qui s'établit entre l'amour éperdu du narrateur pour sa bien-aimée, et l'impatience qu'il manifeste à l'égard de la nature.

Cummins compare le motif de l'introduction de piano avec un passage de l'« Épithalame " de l'opéra Gwendoline (1886) de Chabrier (voir les exemples $2 \mathrm{a}$ et $2 \mathrm{~b}$ ). Cette observation est intéressante car la ligne vocale de Chabrier, outre les similitudes de courbe mélodique et de mètre, se présente aussi comme une monodie à la tonalité ambiguë (l'« Épithalame » commence en sol bémol, mais le passage cité suggère $m i$ bémol mineur). Le lien n'est pas sans équivoque cependant : le duo de Chabrier adopte une carrure un peu martiale (réminiscence de la cabalette des duos d'amour de grand opéra), mais ce n'est pas suffisant pour effacer la pertinence et la suggestivité de l'observation (surtout que l'« Épithalame » est un des moments tournants de Gwendoline, et un des morceaux les plus connus de l'œuvre) ${ }^{14}$. Cummins suggère que la citation symbolise peut-être ironiquement le destin d'un amour condamné, fatalité que partagent le couple de l'opéra de Chabrier et les amants de Verlaine. J’ajoute un parallèle supplémentaire qui peut éclairer le sens poétique du prélude de l'accompagnement de piano de Debussy : le compositeur devait reprendre le même motif au début de son opéra inachevé Rodrigue et Chimène (1890-1893), qui revêt la forme d'une sonnerie de cor, tout de suite après le lever du rideau (voir l'exemple $2 \mathrm{c})^{15}$. Les amants ont convenu qu'à l'arrivée de Rodrigue près de la fenêtre de sa bien-aimée, le jeune homme alerte Chimène en soufflant dans son instrument ${ }^{16}$. La sonnerie de cor n'est pas harmonisée, comme le motif " $\mathrm{x}$ » au début de la mélodie des Ariettes oubliées. Cette citation peut aider à expliquer «Spleen » rétrospectivement : si la mélodie de Debussy essaie d'évoquer une sonnerie de cor par le chant de la main droite du piano, il joue sur deux associations poétiques contrastées de cet instrument : dans « $\mathrm{x}$ », l'instrument suggère la mélancolie de la forêt, la complicité avec la nature, alors que " y » évoque plutôt l'association du cor avec la chasse et le combat, le lien antagoniste à la nature (là où l'homme cherche à dominer son environnement naturel) et le rapport à l'amante perçue comme adversaire, par analogie avec le gibier. Les deux versants de la symbolique du cor correspondent bien aux deux

14 Il n'est pas inutile de clarifier la question de la chronologie, que Cummins n'aborde pas : bien que l'œuvre n'aie pas été représentée à Paris avant 1893 (soit bien après la composition des Ariettes oubliées), elle avait été créée à La Monnaie de Bruxelles dès 1886, publiée cette année-là en version chant et piano, et des extraits avaient été donnés en concert à Paris avant 1893.

15 Debussy a écrit la partie instrumentale sur une particelle et ne l'a jamais orchestrée. Dans l'édition critique publiée chez Durand, le passage apparaît aux mes. 72-76, 78-82 et 115-120. Voir Rodrigue et Chimène de Debussy (2003).

16 Il n'est pas indifférent de noter que les opéras Rodrigue et Gwendoline ont tous les deux été composés sur des livrets de Catulle Mendès. 


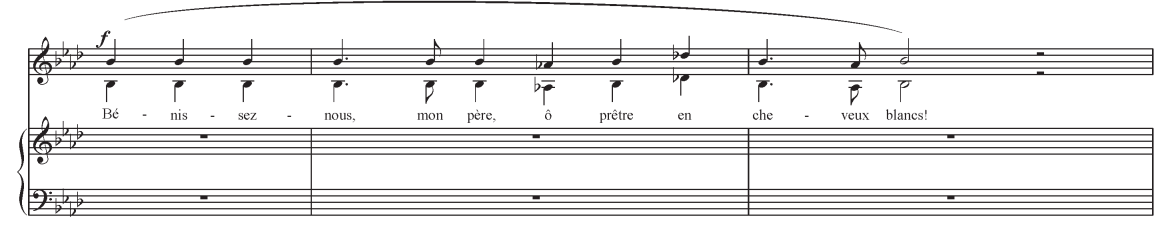

Exemple 2a : Emmanuel Chabrier, extrait de l’«Épithalame» de Gwendoline.

Lent

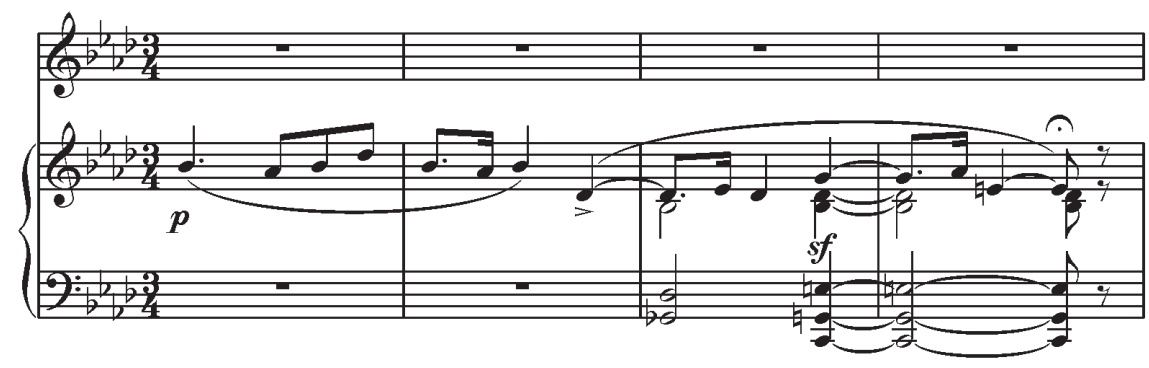

Exemple 2b : Debussy, « Spleen », mes. 1-4.

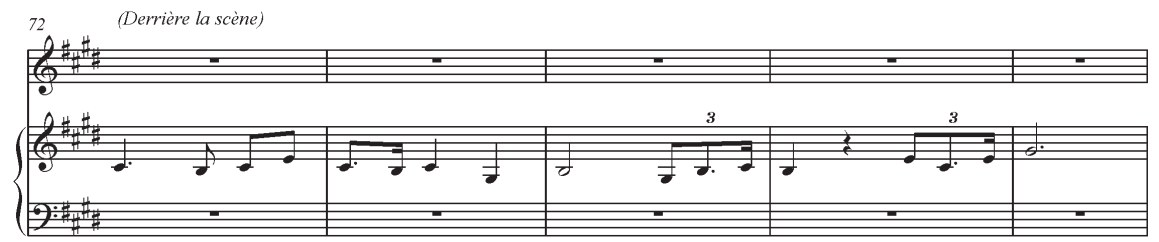

Exemple 2c : Debussy, Rodrigue et Chimène, mes. 72-76.

sentiments qui tiraillent l'esprit du narrateur, avec son amour idolâtre pour l'amante et l'exaspération que lui inspire la nature entière.

Dans son analyse du " Tombeau des Naïades », Cummins cite les travaux de Richard Langham Smith (1989, 107-139) sur Pelléas et Mélisande, qui établissent l'association des tons enharmoniques de $f a$ dièse et sol bémol majeurs avec la lumière et la poursuite de l'idéal. De manière plus générale, ce ton est associé à quelques reprises à l'amour dans des œuvres antérieures à Pelléas : outre le "Tombeau des Naïades " cité par Cummins, c'est déjà le cas dans Diane au bois (1885) (par extension, on peut même plaider son utilisation dans des œuvres instrumentales, comme dans le Quatuor à cordes ou La Mer) ${ }^{17}$. De plus, «l'accord de Tristan », cité dans Pelléas, s’intègre bien au ton de l'amour. Les accords de quinte diminuée et septième mineure sur $f a$ bécarre ou mi dièse ont la fonction de vii 7 dans les tonalités enharmoniques de l'amour ${ }^{18}$, et constituent les quatre notes supérieures de l'accord de V9. Les Ariettes oubliées sont

17 Remarquer que l'«Épithalame » de Chabrier adopte également le ton de sol bémol majeur.

18 Plusieurs auteurs abordent la citation de « l'accord de Tristan » dans l'œuvre de Debussy, dont Carolyn Abbate (1981), David Code (2001 et 2007) et moi-même (2007) (j'exclus ici les travaux portant sur la citation explicite qu'on trouve dans le « Colliwogg's Cake Walk» des Children's Corner). 
écrites durant la phase de wagnérisme intempestif de Debussy, lorsqu'à la villa Médicis il joue à quatre mains des réductions de Wagner avec Paul Vidal, et alors qu'il se rend à Bayreuth (1888) pour entendre les opéras du compositeur allemand. Dans les deux mélodies, le ton de sol bémol et les citations de «l'accord de Tristan » semblent associés à la confession de l'amour.

Dans « Spleen », Verlaine confère à l'expression du sentiment amoureux une forme paradoxale, qui combine le sentiment d'une adoration absolue avec le désagrément qui résulte de l'insécurité qu'elle suscite et de la tyrannie et la servitude qu'elle impose. Dans les derniers vers du texte, le sentiment est décrit avec force et concision. Ils commencent par une progression vers un climax qui coïncide dans le texte avec une déclaration décrivant l'empire de l'amour, dont on mesure la force par l'ampleur du sentiment de rejet qui lui sert de contrepoids, et qui embrasse tout ce qui ne relève pas de la bien-aimée : "Du houx à la feuille vernie,/Et du luisant buis je suis las,/Et de la campagne infinie,/Et de tout, fors de vous [...] ». L'énumération par laquelle commence la phrase procède par amplification (progressant de types de végétaux particuliers, le houx et le buis, à la nature entière). La construction grammaticale débute par un complément d'objet indirect qui retarde l'arrivée du verbe, et ultimement, celle de l'expression de l'amour absolu, conférant ainsi à ce syntagme un impact plus retentissant. Puis tout juste après avoir exprimé ce sentiment, la conclusion de la phrase offre une forme de déni (ou à tout le moins une forme de distanciation désabusée) à la force de l'amour. Un simple « Hélas! » jeté en fin de poème exprime subitement l'aliénation du narrateur, le ressentiment qu'il semble éprouver face à l'emprise despotique d'un sentiment si étouffant.

La réalisation musicale de Debussy exprime brillamment l'effet de cette description verbale, en jouant à la fois sur l'ambiguïté du ton principal du morceau et sur le symbolisme associé à la fois au ton de sol bémol et à "l'accord de Tristan " (voir l'exemple 3). Si on se rend à la mesure finale de «Spleen » pour déterminer le ton principal du morceau, $f a$ mineur s'impose sans ambiguïté. Mais au moment où l'on entend la mes. 28, le cours de la musique comme le récit du poème peuvent laisser croire que la fin est imminente, et la progression harmonique cadentielle en sol bémol au moment du climax et de l'aveu des sentiments amoureux du narrateur laisse croire que la pièce établit finalement le ton de sol bémol majeur. Sur le plan sémantique, le symbolisme de sol bémol (ton de l'amour) est renforcé par l'allusion à «l'accord de Tristan» $\left(\mathrm{T}_{0}\right)$, à la fois mélodiquement, à la main droite du piano, et harmoniquement, dans les notes supérieures de l'accord de V9 (voir le crochet et l'encadré à la mes. 27 de l'exemple). L'amertume du « Hélas! » final du texte est traduite musicalement par la terminaison particulière de la voix sur un fa bémol, en suspens (mes. 29-30). La partie vocale se conclut ainsi sur une note instable par rapport à la tonique supposée de sol bémol, sans résolution. L’effet de déni musical du « Hélas » de la voix est renforcé par la terminaison du piano, à qui revient le soin de résoudre le $\mathrm{fa}$ bémol et de conclure la pièce (à la fois musicalement et narrativement). Le piano expose deux fois le motif monodique du début, " $\mathrm{x}$ ", à chaque fois sur les notes originales (il est identifié par un crochet aux mes. 28-29 et 30-31) : l'harmonisation triomphale de « $\mathrm{x}$ » en sol bémol majeur au moment du climax 
(mes. 28-29) contraste avec la diaphane présentation monodique des mes. 1-2 et sa tonalité ambiguë. Ensuite, la récurrence de " $\mathrm{x}$ » au moment du « hélas » bifurque de sol bémol vers $f a$ mineur, en réinterprétant la note conclusive de $f a$ bémol à la voix en $m i$ bécarre (voir les tirets obliques sur l'exemple) ${ }^{19}$. Ainsi, dans l'esprit du fragment, et bien que la pièce se termine par une cadence claire en $f a$ mineur, les huit dernières mesures jouent sur l'ambiguité de l'établissement de la tonalité, au même titre que le début. Et cette ambiguïté est également liée au sens profond du poème : le tiraillement du narrateur entre l'amour éperdu qui l'habite (ton de sol bémol majeur) et le sentiment d'aliénation qui l'accompagne (ton de $f a$ mineur du « Hélas » final).

Le sens du début hors tonique de «Spleen » s'approfondit encore si on le situe dans le contexte de la fin du mouvement précédent. Les mes. 3-4 de «Spleen » exploitent l'ambiguité d'une progression qui peut s'interpréter dans le ton de sol bémol ou de $f a$ (comme l'indique le chiffrage harmonique sous la portée dans l'exemple 3) : on peut analyser les deux accords à distance de triton selon la tonalité de sol bémol si, suivant la convention, on considère qu'ils commencent par un accord de tonique, suivi d'un accord non fonctionnel et contrastant de $d o$. Ou encore, on peut analyser le début en $f a$ mineur, avec une progression incomplète (privée de tonique initiale ${ }^{20}$, par l'enchaînement ${ }^{b}$ II-V9.

Comme le fait finement remarquer Cummins, l'interprétation d'une progression en sol bémol s'inscrit naturellement dans la résonance de la fin de la mélodie précédente, "Green », qui conclut clairement dans cette tonalité. Le ton de l'amour sert en quelque sorte de lien entre les deux mélodies :

\begin{tabular}{|c|c|c|c|c|}
\hline \multirow[b]{2}{*}{ "Green » : } & Début ambigu & Cadence finale claire & & \\
\hline & $\begin{array}{l}\text { la bémol : i ? } \\
\text { Sol bémol : ii ? }\end{array}$ & Sol bémol : I & & \\
\hline \multirow{2}{*}{\multicolumn{3}{|c|}{ "Spleen » : }} & Début ambigu & Cadence finale claire \\
\hline & & & $\begin{array}{l}\text { Sol bémol : I? } \\
\text { fa mineur : }{ }^{b} \text { II ? }\end{array}$ & fa mineur : $\mathrm{i}$ \\
\hline
\end{tabular}

Si on aborde «Green " plus en détail maintenant, la mélodie semble faire également allusion au Tristan und Isolde de Wagner et exploiter le symbolisme du ton de l'amour. Ici, l'observation de Cummins sur l'effet in media res est particulièrement frappante : la mélodie débute sur une harmonie ambiguë, qui semble d'abord s'interpréter comme la tonique de la bémol mineur, mais se révèle rétrospectivement assurer une fonction de ii en sol bémol majeur. Par son ambiguïté initiale et sa réinterprétation comme début hors tonique, l'harmonie initiale transpose de manière très suggestive l'effet du début in media res du texte, qui met en scène la brusquerie d'un poète troublant le réveil de

19 L'œuvre revient en fait à l'interprétation du mi bécarre (dans un accord de do) qui avait été proposée dès la première manifestation du motif " $\mathrm{y}$ " aux mes. 3-4, puis à nouveau aux mes. 12-13 et 20. Mais la résolution sur l'accord de $f a$ mineur, qui avait été constamment évitée, se réalise finalement pour la première fois à la toute fin de l'œuvre, aux mes. 33-34. L'évitement répété de la tonique, dont l'arrivée est repoussée le plus loin possible, rappelle l'écriture du Tristan und Isolde de Wagner.

20 Une autre mélodie du même recueil des Ariettes oubliées, "L'Ombre des arbres ", débute également avec deux accords placés à distance de triton. 


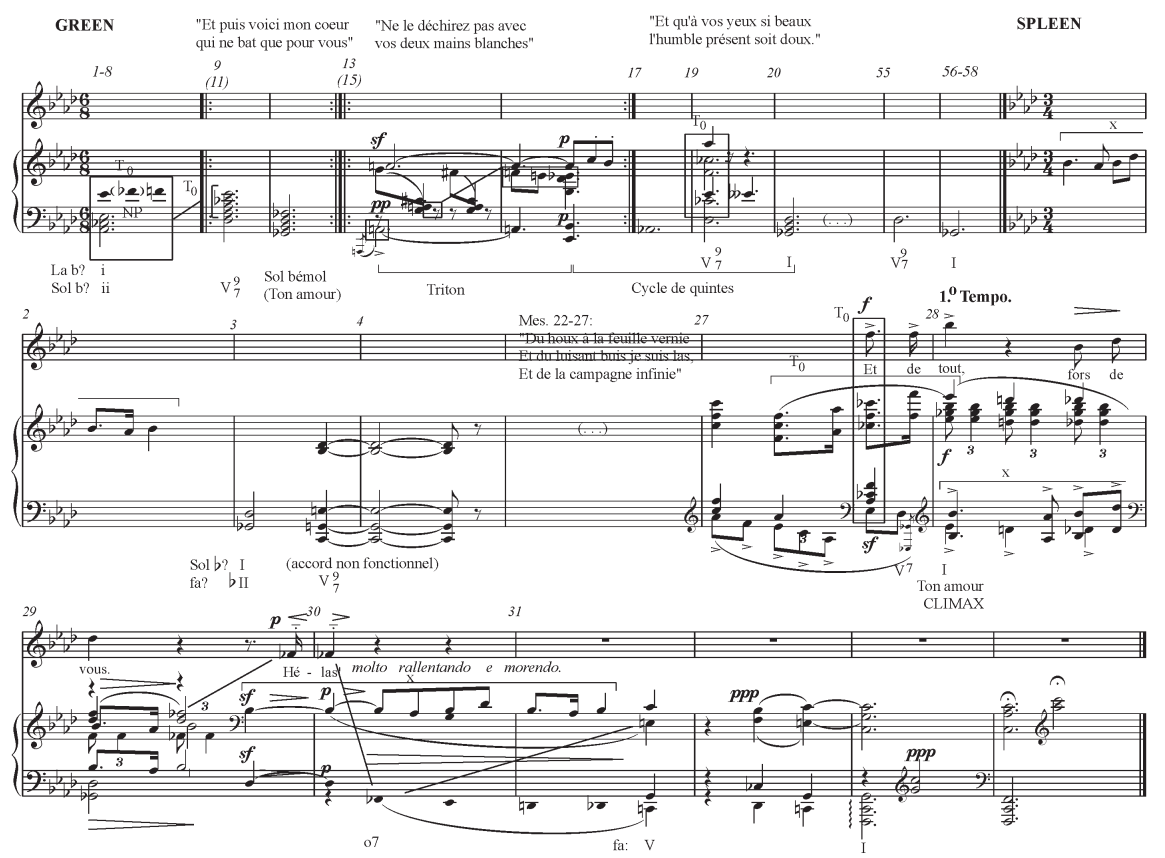

Exemple 3 : Réduction de passages stratégiques de « Green » et «Spleen ».

sa bien-aimée par le don d'une offrande et par une déclaration d'amour intempestive, et dont l'arrivée du dehors est révélée seulement après coup. Si on $\mathrm{y}$ inclut le $f a$, les huit premières mesures de l'œuvre prolongent un accord qui reprend par enharmonie les mêmes notes que "l'accord de Tristan ", noté " $\mathrm{T}_{0}$ " sur l'exemple (les notes de sol dièse-si-ré dièse-fa bécarre de l'accord de Wagner sont réécrites sous la forme de la bémol-do bémol-mi bémol-fa) - le fa bémol constitue une note de passage chromatique. La résolution sur un accord de sol bémol, qui s'impose rétrospectivement comme la tonique et symbolise le ton de l'amour, coïncide à chaque fois avec un épanchement amoureux. La première fois, sur les mots «Et puis voici mon cour qui ne bat que pour vous » (mes. 9-10 et 11-12), la résolution est affaiblie par la présence d'une septième mineure dans l'accord de sol bémol. La deuxième fois, l'offrande de la cueillette et du cœur du poète sur les paroles «Et qu'à vos yeux si beaux/l'humble présent soit doux » est associée à la première progression cadentielle claire de la pièce (mes. 17-20), fondée sur un cycle de quintes. Cet enchaînement intègre l'accord $\mathrm{T}_{0}$ à une harmonie de ré bémol neuvième de dominante (encadrée à la mes. 19) et se résout sur la tonique de sol bémol.

L'enchaînement harmonique conventionnel de la progression cadentielle est précédé d'une succession instable de deux accords à distance de triton, des accords de septième de la bécarre et mi bémol (mes. 13-16). La tension harmonique souligne l'inquiétude du narrateur ("Ne déchirez pas [mon cœur] avec vos deux mains blanches »), et on peut y déceler une allusion au prélude de Tristan. En effet, les voix intérieures font entendre le tout début du prélude de l'opéra 
de Wagner, le mouvement mélodique qui approche $\mathrm{T}_{0}$ (la-fa-mi-ré dièse chez Wagner, dont la dernière note est réinterprétée sous la forme d'un mi bémol chez Debussy, voir les encadrés des mes. 13-16).

\section{CANOPE}

Une autre analyse de Cummins pose d'intéressants problèmes d'interprétation de la syntaxe musicale, exploitant une ambiguïté telle qu'elle semble remettre en cause la possibilité d'une organisation structurelle univoque. L'auteure analyse le prélude pour piano Canope pour illustrer la fin ouverte. L'œuvre, qui adopte une tonalité progressive, débute dans le ton de ré modal et se termine sur un accord de $d o$, à la polarité ambiguë. On peut également considérer que cette pièce illustre le genre du fragment dans deux autres sens : d'abord, par le caractère disparate du matériau musical - ce prélude fait preuve d'une abondance étonnante de matériau pour une si courte pièce, et ses diverses composantes emploient aussi des styles très contrastés ; ensuite, Canope illustre également le genre du fragment par la manière dont l'organisation harmonique et tonale de la pièce repose non sur l'expression de repères clairs (qu'ils soient stables ou mouvants), mais sur leur ambiguïté.

Cummins rend compte de nombreuses analyses, aux méthodologies ou perspectives différentes : par exemple, Serge Gut (1980) montre l'importance des accords formés de quintes superposées (comme do-sol-ré) - agrégats irréductibles aussi bien aux accords de tierces superposées qu'aux « set classes " (parce que leur identité auditive repose sur leur disposition intervallique, qui ne peut être nivelée comme dans un "set class»). Ou encore, Thomas Warburton (1982) interprète la structure en fonction de la compétition pour la prééminence du ré éolien et du do mixolydien, lutte responsable d'effets quasi polytonaux. Cummins fournit sa propre interprétation, légitime, mais similaire aux autres dans la mesure où elle cherche à dégager une interprétation univoque et définitive ${ }^{21}$. L'analyse que je propose ici reprend des éléments d'interprétation proposés par Cummins et d'autres théoriciens, mais je suggère qu'une étude plus fidèle au genre du fragment développe une interprétation plurielle de la structure, où l'ambiguïté du sens est considérée comme irréductible. En d'autres termes, l'esprit du fragment s'applique non seulement aux éléments de la pièce elle-même (assemblage composite de fragments, accumulation de matériau hétéroclite), mais également à leurs rapports (polysémie de la syntaxe qui lie ces éléments). En ce sens, Canope développe une forme d'organisation fragmentaire inconnue de "Green » et de «Spleen».

Dans l'exemple 4 ci-dessous, je reprends les lettres « $U$ » $\mathrm{à}$ $Z$ » de Cummins pour désigner les six motifs caractéristiques qui composent le matériau disparate de l'œuvre (la division de l'exemple en trois systèmes, $4 \mathrm{a}$, b et c, est discutée plus en détail ci-dessous). Dans la pièce, « $U$ », «V » et « $\mathrm{W}$ » sont traités à la manière de ce que Jean Barraqué appelle des «thèmes-objets » : bien qu'ils

${ }^{21}$ Voir la discussion de la p. 93 et le graphe très stimulant de la p. 94. Le commentaire aurait pu être plus développé, entre autres pour expliquer l'interprétation d'un accord de si bémol structurel à la mes. 17, que j'avoue ne pas comprendre. 
se présentent dans des contextes différents, ils sont repris à chaque fois sur les mêmes notes, à quelques légères variantes près ${ }^{22}$. Ce procédé témoigne d'un rejet du développement classique, et renforce l'impression de fragmentation du discours (un peu comme ce qu'on retrouve dans la musique de Stravinski). « U » (aux mes. 1-5 de l'exemple 4a) se subdivise lui-même en éléments contrastés, chaque fois bien circonscrits par leur phrasé. « U1 » propose une mélodie "épaissie » par une série de triades parallèles ${ }^{23}$. Dans « $\mathrm{U}_{2}$ », une ligne de basse est introduite sous la ligne d'accords, et l'organisation tonale des deux parties semble évoluer de manière indépendante. " $\mathrm{U}_{3}$ " revient à une texture homorythmique, mais en produisant des mouvements indépendants entre les lignes de soprano et de basse des accords (du moins à partir du deuxième accord). C'est à ce moment que l'arrivée du ré se produit dans la structure harmonique profonde, grâce à l'introduction du registre grave et à l'effet cadentiel au début de la mes. 5 .

Une vue d'ensemble suggère que la pièce adopte une coupe ternaire traditionnelle, car le matériau des mes. 1-5 est repris aux mes. 26-30 avec quelques altérations, en débutant sur le même pôle de ré qu'au début. Cependant, trois déterminants de l'organisation structurelle remettent en cause cette division tripartite et produisent une ambiguité formelle : le retour du pôle de ré n'est pas assez solidement établi pour s'imposer dans la structure profonde de l'œuvre, le cours du thème bifurque ensuite de telle manière que la mes. 30 cadence hors de ré, et l'œuvre se termine sur une harmonie ambiguë. Un effet de parallélisme motivique se produisant sur plusieurs niveaux structurels de l'œuvre a partie liée avec cette ambiguïté formelle, et il est nécessaire d'en rendre compte avant de décrire plus en détail l'ouverture de la forme et la polysémie de la syntaxe de Canope.

La séquence d'accords construits sur les fondamentales ré-sol-do fait l'objet d'un déploiement sur plusieurs niveaux structurels ${ }^{24}$. À la surface de l'œuvre, elle se manifeste sur les trois derniers temps de la mes. 2 (identifiés par un crochet sous la portée supérieure de l'exemple 4a). Mais on la retrouve sur trois niveaux de structure de plus en plus profonds, associés aux trois systèmes de l'exemple 4 (notés $\mathrm{a}, \mathrm{b}, \mathrm{c}$ ), où elle est identifiée dans chaque cas par un crochet sous la portée. Dans l'exemple 4a, la séquence de fonctions sous-tend le début de la première phrase : le matériau " $\mathrm{U}$ » commence et se termine en ré, mais le fragment « $\mathrm{U}_{2}$ » (mes. 3-4) articule la ligne de basse sol-do ${ }^{25}$. Dans l'exemple 4 b, la séquence initiale est projetée à un niveau plus profond à l'aide d'une

22 Pour une définition du thème-objet, voir Barraqué $(1962,154)$. Pour une discussion critique de l'application de ce concept à la musique de Debussy, voir Laurence Berman $(1965,217)$ et de Médicis (2007).

23 J'emploie le terme « épaissie » de préférence à « harmonisée », car il ne s'agit pas d'accords fonctionnels.

24 En anglais, « enlargment » constitue un cas particulier du procédé plus général du « parallélisme motivique " (ou " motivic parallelism »), pour reprendre l'expression de Charles Burkhart. Voir Burkhart (1978), « Schenker's Motivic Parallelisms », et Brian Alegant et Don McLean (2001).

25 En fait, la progression de la mes. 3-4 s'avère problématique sur plusieurs points, ne serait-ce qu'en raison du rapport du mouvement de la basse et des accords de la main droite, qui semblent étrangers l'un à l'autre. Je reviendrai plus tard sur cette question en rapport avec l'exemple 5. 


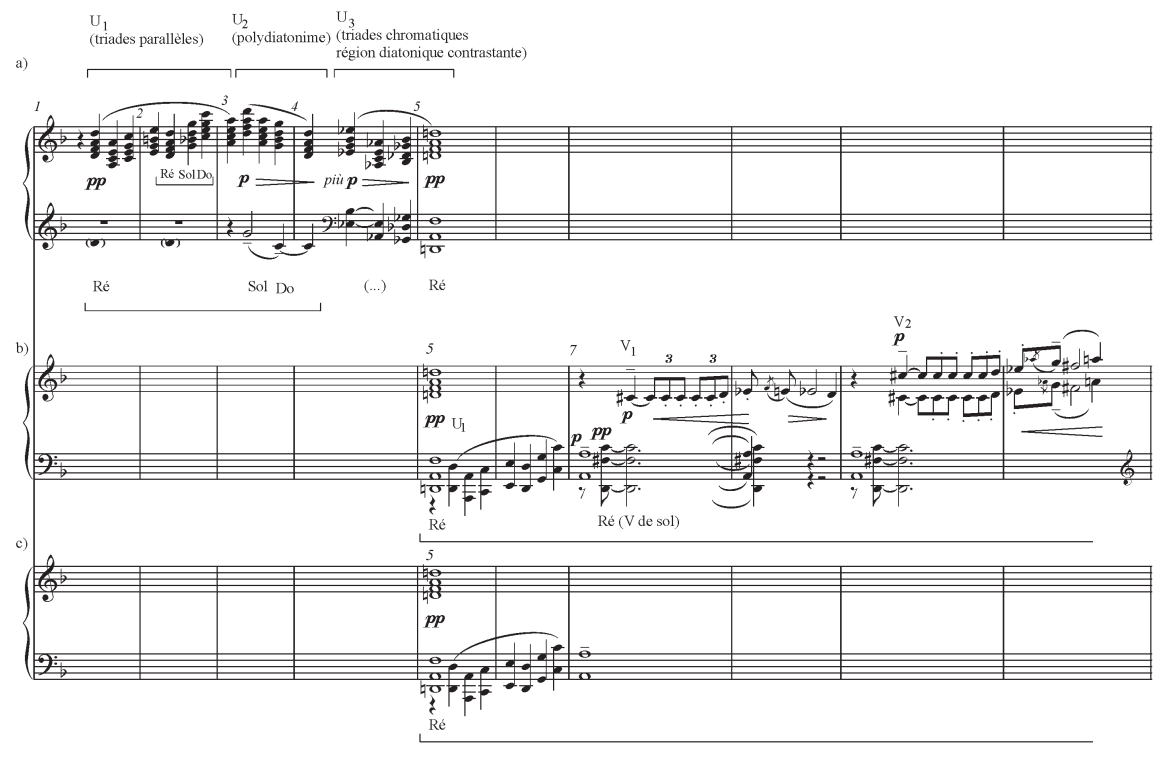

a)

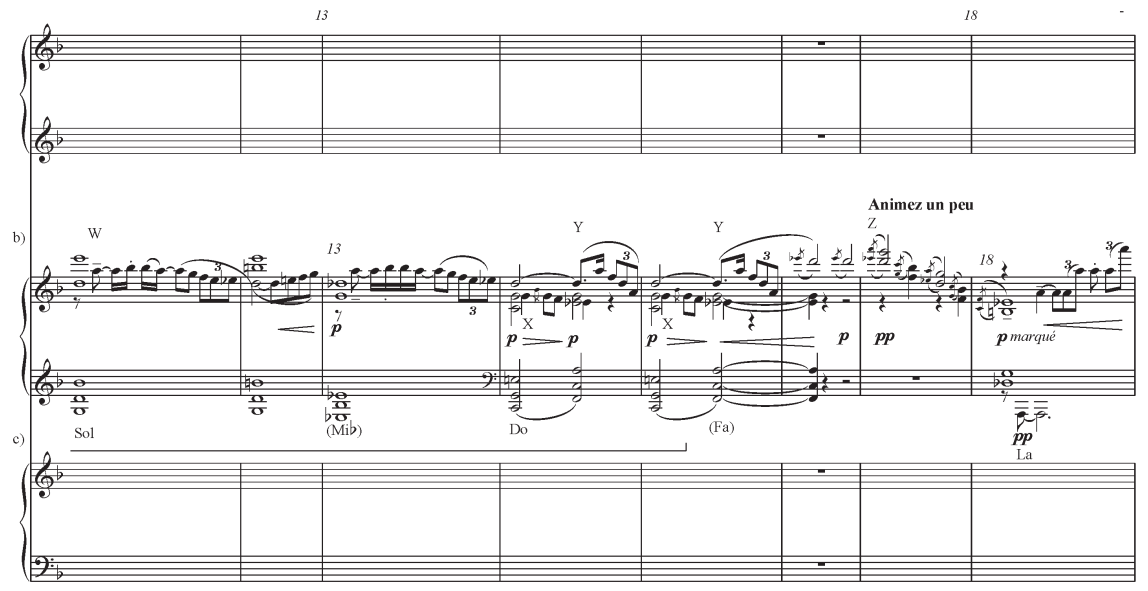

Exemple 4 (page 1 à 4): Canope. 

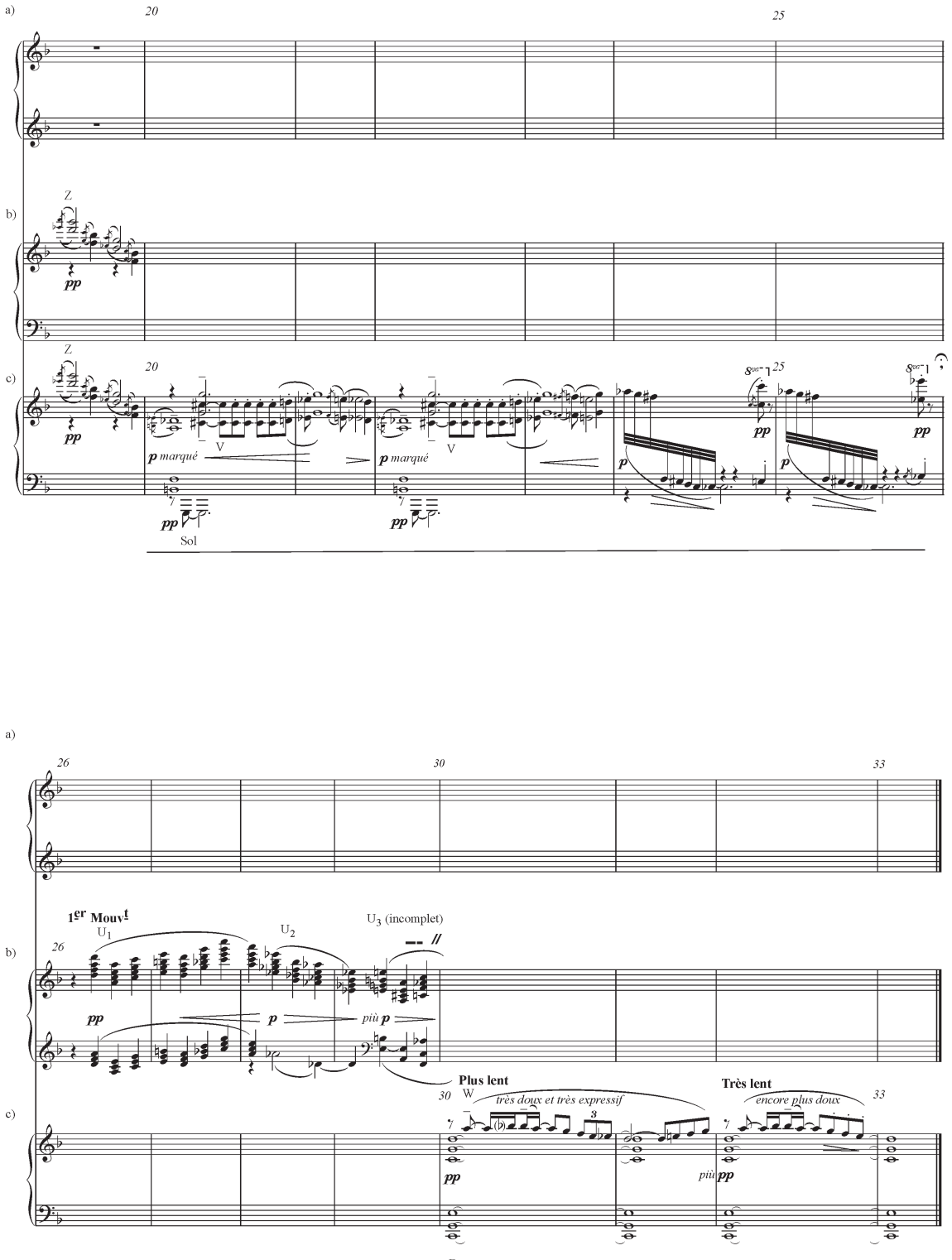
progression d'accords par quintes descendantes : Ré-Sol-Do-Fa. À la mesure 13, l'accord de $m i$ bémol qui sépare le $s o l$ du do à la basse crée un effet d'arpégiation descendante.

Pour le passage qui couvre la mes. 18 jusqu'à la fin de la pièce, on observe une forme d'interférence, de contradiction entre les interprétations des deux niveaux les plus profonds (exemples $4 \mathrm{~b}$ et $4 \mathrm{c}$ ). Dans une petite pièce ternaire tonale, la deuxième section se termine généralement sur la dominante (souvent pour produire ce qu'on appelle en langage schenkérien une interruption), suivi du retour du matériau initial dans le ton principal, pour conclure dans cette tonalité. On peut déceler une allusion à une récapitulation traditionnelle à la mes. 26, désamorcée de deux manières aux mes. 20-25 et 29-33. Commençons par la fin : lorsque le thème " $U$ » est repris à partir de la mes. 26, seul le début de ce passage suggère un pôle de ré (dans le registre aigu, avant que la basse ne soit clairement articulée). Au moment d'établir la tonique par une cadence dans le registre grave aux mes. 29-30 (le passage correspondant aux mes. 4-5, avec son mouvement indépendant de la basse), la musique bifurque vers do. La récapitulation est donc désamorcée par l'absence de confirmation cadentielle du pôle de ré. Le second effet déstabilisant se produit aux mes. 20-25: peu avant, la mes. 18 semble d'abord annoncer l'arrivée imminente de la section A' par la liquidation du matériau motivique (produite par le matériau peu différencié « $\mathrm{Z} »)^{26}$ et l'établissement de l'harmonie de la, dominante du ton initial (avec un accord de $\mathrm{V}^{9}$ et quinte abaissée). Mais quand le même matériau est repris deux mesures plus loin, l'harmonie a été transposée à la seconde inférieure sur un accord de sol, dominante de do. C'est donc comme si la dominante du ton initial de ré était rectifiée par la dominante de $d o$, annonçant le pôle conclusif (ou de manière plus juste, la basse conclusive). Ensuite, le sol est laissé en suspens, la fausse récapitulation qui se cantonne dans le registre aigu agit comme une parenthèse, puis la préparation déstabilisante des mes. 20-25 se résout après la parenthèse, avec le rétablissement du registre grave sur le do des mes. 30-33. On remarque que le matériau « $\mathrm{V}$ » assume toujours une fonction similaire, étant placé à chaque fois sur la dominante d'un des accords structurels de la séquence ré-sol-do : à la mes. 7, il introduit le sol dans l'expansion de niveau intermédiaire (exemple 4b), et à la mes. 20 , il introduit le do du niveau profond (avec une ligne mélodique utilisant toujours à peu près les mêmes notes, malgré le changement d'accords).

L'ambiguïté qui affecte l'organisation à grande échelle s'exerce non seulement sur le plan des pôles, mais également sur celui du type de langage harmonique (polydiatonisme, monotonalité, harmonie triadique, harmonie par quintes superposées). Et cette équivoque harmonique se manifeste également à la surface, dès la présentation du matériau du début (voir l'exemple 5). Un choc se produit dans « $\mathrm{U}_{2}$ » entre les triades de la main droite et le mouvement mélodique de quinte descendante à la main gauche. Cela suggère différentes interprétations, qui n'ont peut-être pas toutes la même prégnance lors de cette

26 Bien que j’aie repris la lettre que Cummins attribue à « $\mathrm{Z}$ », il est préférable de le considérer comme un matériau indistinct, et non comme une véritable entité motivique ou thématique. 
première manifestation, mais qui se réverbèrent toutes au cours de la pièce. L'exemple 5 propose trois interprétations différentes : $5 \mathrm{a}$ montre comment la combinaison du mode de ré éolien suggéré à la strate supérieure et du pôle de do défini par le mouvement de basse de la strate inférieure produit une forme de polydiatonisme ${ }^{27}$. Les exemples $5 \mathrm{~b}$ et $5 \mathrm{c}$ identifient différentes structures intervalliques implicites dans la succession d'accords, dont l'importance devient plus évidente par la suite : il s'agit d'une part des accords de quintes superposées (voir les encadrés dans l'exemple 5b), des sous-ensembles des verticalités qui se produisent dans ces mesures, et d'autre part de l'accord de neuvième (exemple $5 c)^{28}$. On remarque que les notes du deuxième accord de quintes superposées correspondent à celles de la séquence génératrice de l'exemple 4, ré-sol-do.

Mais ces interprétations selon les accords de quintes superposées et selon des neuvièmes entrent en contradiction l'une avec l'autre, et conjointement, elles s'opposent logiquement à l'interprétation polydiatonique. D'une part, les accords par quintes superposées sont des " objets sonores " ayant leur physionomie propre, et ce serait les dénaturer que de les assimiler à des accords de neuvièmes incomplets (accords qui dérivent d'une logique de superposition de tierces). D'autre part, l'intégration des notes de la main gauche (portée inférieure de l'exemple 5a) et celles de la main droite au sein d'un seul accord, qu'il s'agisse de quintes superposées ou d'accords de neuvième, est antinomique avec une conception polydiatonique, car elle gomme l'hétérogénéité des deux strates. C'est pour être cohérent avec une interprétation polydiatonique que, dans l'exemple 5a, je propose de désigner les deux verticalités encadrées comme «Ré sur Sol » et "Ré sur Do », et non par exemple comme un accord de neuvième de sol incomplet (sol-[...]-ré-fa-la) et un accord de septième de ré en troisième renversement - j'ai sélectionné spécialement ces deux verticalités car plus loin elles se déploient à un niveau plus profond. Bien que ces trois interprétations soient théoriquement irréductibles les unes aux autres, elles s'affirment toutes au plan structurel dans la pièce, et j'avance que l'originalité de Canope réside ainsi non seulement dans l'éclatement des éléments du discours musical, sensible dans l'hétérogénéité des fragments « $\mathrm{U}$ » $\mathrm{a}$ « $\mathrm{Z}$ », mais également dans les interprétations contradictoires que l'interaction de ces éléments engendre, et que le discours musical force à considérer conjointement.

Tout comme on observe le déploiement de la séquence ré-sol-do sur différents niveaux structurels, de même les différentes interprétations associées aux mes. 3-4 (illustrées dans l'exemple 5) font l'objet d'une transposition à un niveau plus profond aux mes. 11-15 (voir l'exemple 6). L'organisation de la mélodie de la portée supérieure de l'exemple $6 \mathrm{~b}$ suggère un pôle de ré (comme la main droite de l'exemple 5a) : le segment «W » établit l'ambitus de quinte la-ré, et

27 Plutôt que de parler de superposition de tonalités (ou de polytonalité), je préfère désigner la combinaison de matériaux diatoniques contrastants (accords, lignes mélodiques) par le terme plus neutre, et plus précis à mon sens, de polydiatonisme. Je discute plus en détail cette notion et ses rapports avec la polytonalité dans « La polytonalité selon Darius Milhaud : 'Plus subtile dans la douceur, plus violente dans la force...' " (2005). Warburton (1982) aborde aussi quelques effets de polydiatonisme dans Canope et d'autres œuvres de Debussy.

28 Comme je l'ai mentionné plus haut, Gut (1980) aborde ce phénomène dans son article « $\mathrm{Ca}$ nope de Debussy, analyse formelle et structure fondamentale ». 


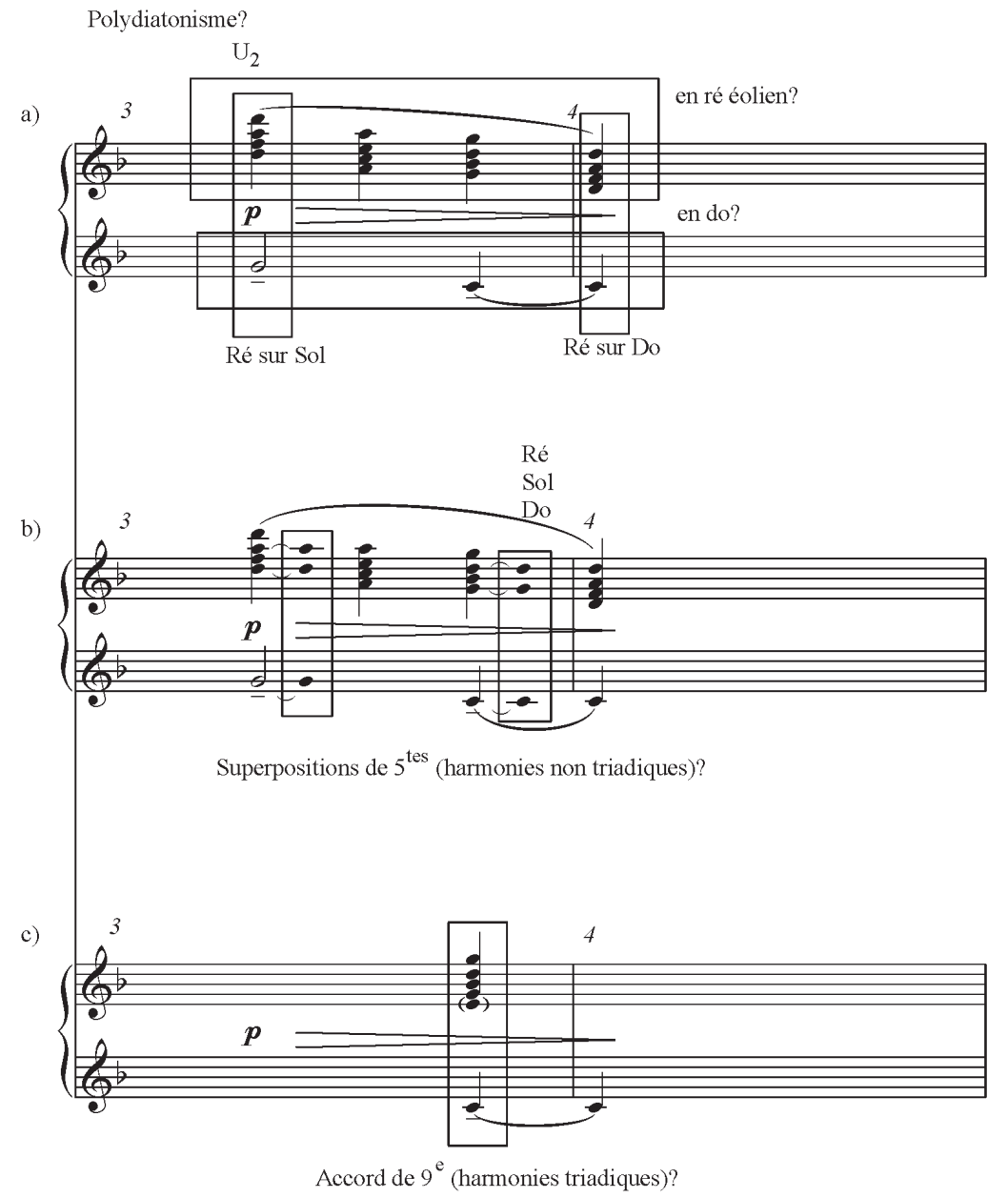

Exemple 5: Canope, mes. 3-4.

le segment « $\mathrm{Y}$ » est fondé sur un arpège de la triade de ré. Cette mélodie se déroule sur des harmonies triadiques de sol et do à la basse, comme l'indique le crochet sous la portée inférieure (une amplification de la main gauche de l'exemple 5a, où le $m i$ bémol de la mes. 13 constitue un élément d'arpégiation, comme je l'ai discuté plus haut). Ainsi, le conflit entre la mélodie et les accords dans l'exemple $6 \mathrm{~b}$ (respectivement la portée supérieure et les deux portées inférieures) suggère une écriture polydiatonique similaire à celle des deux strates de l'exemple 5a. De plus, on retrouve la même ambiguité entre les différentes formes d'organisation accordique que dans l'exemple 5. Les encadrés de l'exemple 6b identifiés par les combinaisons de "Ré sur Sol » et "Ré sur Do » correspondent à la projection à un niveau plus profond des entités analogues encadrées sur l'exemple 5a. Les effets d'accords de quintes superposées et d'accords de neuvième, qui restaient à l'état latent dans les mes. 3-4, s'affirment beaucoup clairement aux mes. 11-15. Les accords en rondes et en noires sans 
hampes dans l'exemple 6a renvoient respectivement aux étagements de quintes et aux accords de neuvième. Les notes sont représentées dans leur registre exact, ce qui démontre que les accords en rondes produisent l'étagement littéral d'intervalles de quinte, du bas vers le haut. Les accords de neuvième sont tous formés de triades majeures avec septième mineure et neuvième majeure (ce qui correspond sur le plan intervallique à des neuvièmes de dominante, sans en assumer la fonction tonale cependant).

L'exemple 7 montre que la conclusion de l'œuvre ne propose pas uniquement le terme ultime de l'augmentation de la séquence ré-sol-do, avec sa terminaison hors de la tonique, mais qu'elle reprend la même ambiguïté harmonique et tonale observée auparavant : l'opposition entre la mélodie en ré de l'exemple $7 \mathrm{a}$ et le pôle de do dans les exemples $7 \mathrm{~b}$ et $7 \mathrm{c}$ suggère le bidiatonisme (comme dans les exemples 5 et 6 ). L'exemple $7 \mathrm{~b}$ suggère deux structures harmoniques concurrentes : d'une part, une superposition de quintes, qui se prolonge vers l'aigu avec l'ambitus ré-la de la portée supérieure ; et d'autre part, une harmonie triadique de $\mathrm{V} 9$, dont le ré supérieur, conjointement avec le mi grave de l'exemple $7 \mathrm{c}$ et le si bémol aigu de l'exemple $7 \mathrm{a}$, assurent respectivement les fonctions de neuvième, tierce et septième par rapport à la fondamentale de do - la fin apparemment abrégée du motif « W » à la mes. 32, non pas sur un ré mais sur un $m i$ consonant avec basse de $d o$, semble renforcer l'interprétation triadique $^{29}$.

Il pourra sembler paradoxal de s'intéresser à l'augmentation structurelle (" enlargment») de la séquence ré-sol-do sur plusieurs niveaux structurels dans une pièce si disparate, si hétéroclite. Mais si l'analyse réductionnelle pratiquée

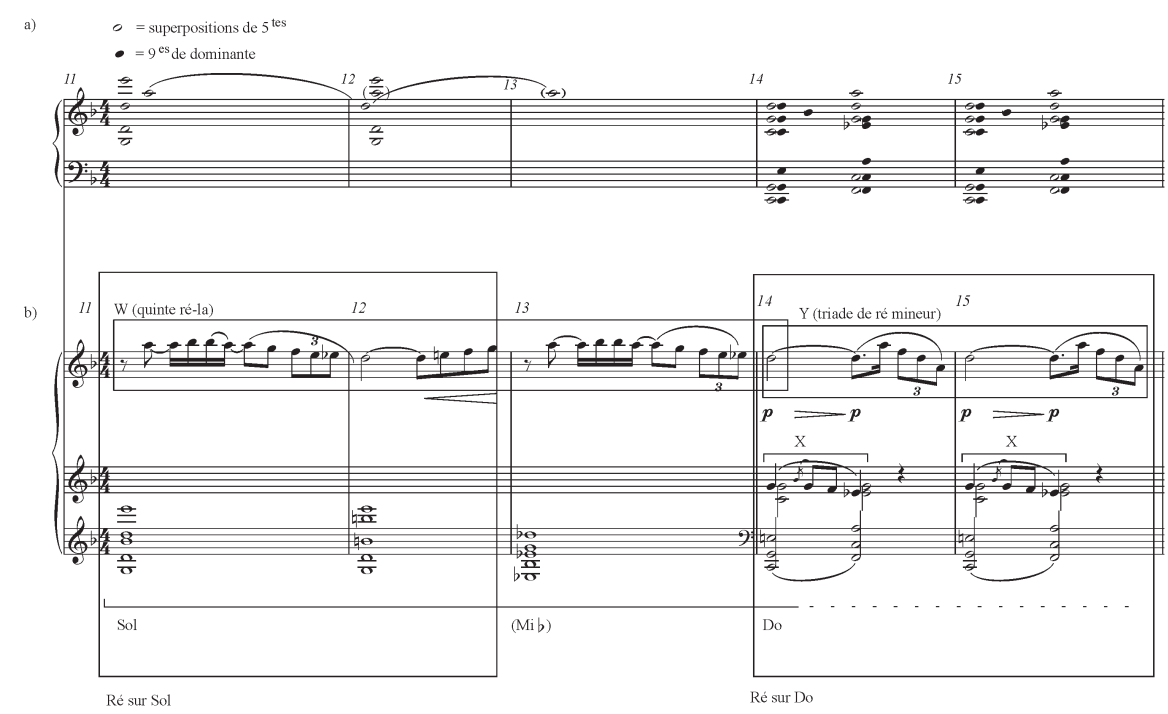

Exemple 6 : Canope, mes. 11-15.

29 Dans l'interprétation en fonction d'un accord de $\mathrm{V}^{9}$, le si bémol de l'exemple 7a est considéré comme une note intégrante de l'accord, réinterprétant le la précédent comme une note ornementale. 


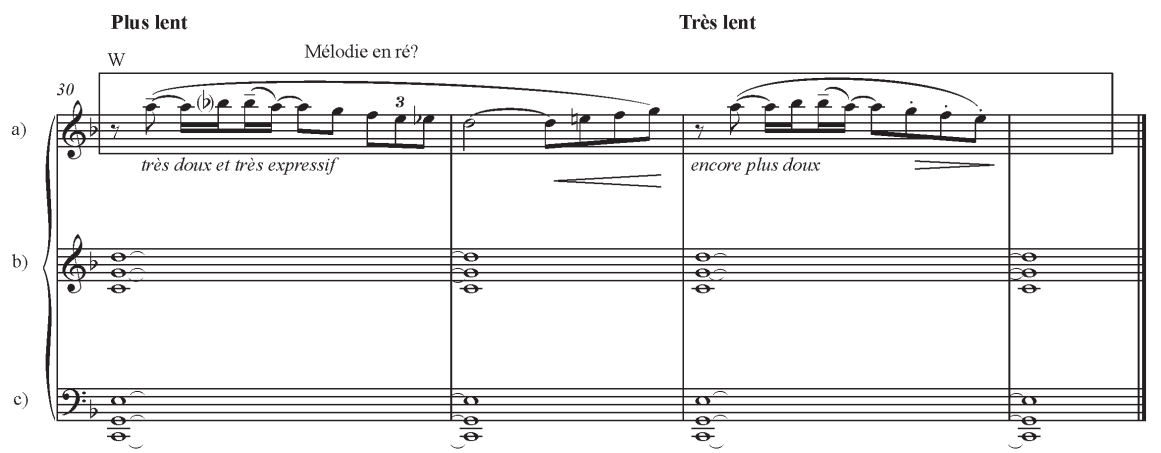

Exemple 7 : Canope, mes. 30-33.

à l'origine par Schenker relevait d'une pensée incontestablement organiciste, cela ne veut pas dire que la transposition de cette méthode et de certains procédés qui en sont issus (comme l'amplification structurelle, ou enlargment en anglais) relèvent nécessairement d'une telle conception. Canope en est un bel exemple : l'œuvre adopte d'une part une structure hiérarchique de l'organisation musicale, où une séquence d'accords donnée se déploie sur de multiples niveaux ; et d'autre part, l'œuvre témoigne d'une conception ouverte par l'absence de résolution tonale (avec sa terminaison sur une sonorité ambiguë), et de manière plus radicale encore, par le caractère polysémique de la syntaxe des hauteurs, aux structures harmoniques s'analysant concurremment selon des principes irréductibles les uns aux autres (harmonies triadiques, accords de quintes superposées, structures polydiatoniques), sans que l'un arrive à dominer les autres.

Le survol de Debussy and the Fragment a permis d'examiner quelques analyses sensibles d'œuvres de Debussy proposées par Linda Cummins. De manière plus générale, nous avons vu que l'ouvrage jette un nouvel éclairage sur le rapport de l'écriture et du style du compositeur français avec son époque et avec ses prédécesseurs, en précisant les liens qu'il entretient avec des œuvres représentatives du fragment musical romantique (chez Chopin et Schumann par exemple). En s'appuyant sur une connaissance approfondie du fragment en études littéraires, Linda Cummins offre de nouvelles clés pour aborder les parallèles entre musique, littérature et arts visuels. S’autorisant de son exemple, on pourrait facilement étendre le filet de l'étude du fragment pour sonder de nouvelles mers (on pense en particulier aux répertoires des $\mathrm{XX}^{\mathrm{e}}$ et $\mathrm{XXI}^{\mathrm{e}}$ siècles). Qu'on ne s'y trompe pas, cela a déjà commencé, comme en témoignent de nombreux travaux consacrés à des figures aussi diverses que Janáček, Schoenberg, Nono ou Kurtág. En fait, les musicologues n'ont pas attendu la vogue de l'étude du fragment en musique pour aborder ce phénomène sous d'autres vocables. C'est le cas de Glenn Watkins (1994), dont Cummins cite les travaux sur le collage (ratissant large, de Stravinski à Schnittke, de John Adams à Krenek). Et il faudrait allonger la liste pour citer les travaux qui portent sur des principes 
esthétiques apparentés tels que l'œuvre ouverte ou le rejet de la temporalité téléologique. En fait, si l'élargissement du corpus abordé dans les études sur le fragment est d'ores et déjà acquis, un danger me semble guetter cette démarche. Et c'est le même qui a affligé l'étude de l'organicisme, un concept qui a connu un tel engouement que dans ses pires excès, l'analyse myope du procédé prenait le pas sur l'évaluation de sa signification et sur l'examen des modalités de ses manifestations (pour une critique fort pertinente de ces débordements, voir Leonard B. Meyer 1991). Par réaction, n'y a-t-il pas à craindre que, maintenant, on soit tenté de célébrer de façon trop simpliste la discontinuité du discours, l'ouverture du sens, l'écriture en mosaïque comme autant de signes irréfutables d'audace, d'originalité et de modernisme ? Sur ce plan, le livre de Cummins est exemplaire : ses discussions évitent les conclusions hâtives et réductrices, et elles maintiennent un bel équilibre entre l'analyse musicale d'œuvres représentatives de l'esthétique du fragment debussyste et l'exploration des filiations esthétiques contextuelles (comme lorsqu'elle observe les rapports de Debussy avec l'arabesque mallarméenne, ou avec la tradition du prélude pour clavier). Il y a fort à parier que cette orientation, qui cherche à affiner l'étude des techniques du fragment, à les contextualiser, à tracer leurs filiations, et à décrire la variété de leurs effets esthétiques, offre encore des perspectives de recherche riches et prometteuses.

\section{RÉFÉRENCES}

Abbate, Carolyn. 1981. "Tristan in the Composition of Pelléas ». $19^{\text {th }}$ Century Music 5, $\mathrm{n}^{\mathrm{O}} 2: 117-141$.

Alegant, Brian et Don McLean. 2001. "On the Nature of Enlargement ». Journal of Music Theory $45, \mathrm{n}^{\mathrm{0}} 1: 31-71$.

Barraqué, Jean. 1962. Debussy. Paris : Seuil.

Berman, Laurence. 1965. The Evolution of Tonal Thinking in the Works of Claude Debussy. Thèse de doctorat, Harvard University.

Burkhart, Charles. 1978. "Schenker's Motivic Parallelisms ». Journal of Music Theory $22, \mathrm{n}^{\mathrm{O}} 2: 145-75$.

Code, David. 2007. «Debussy's String Quartet in the Brussels Salon of 'La Libre Esthétique'». $19^{\text {th }}$ Century Music 30, $\mathrm{n}^{\mathrm{O}} 3: 257-287$.

—. 2001. «Hearing Debussy Reading Mallarmé: Music après Wagner in the Prélude à l'après-midi d'un faune ». Journal of the American Musicological Society $54, \mathrm{n}^{\mathrm{O}} 3:$ : 493-554.

Daverio, John. 1993. Nineteenth-Century Music and the German Romantic Ideology. New York : Schirmer Books ; Toronto : Maxwell Macmillan Canada ; New York : Maxwell Macmillan International.

Debussy, Claude. 2005. Correspondance, 1872-1918, éditée par François Lesure, Denis Herlin et Georges Liébert. Paris : Gallimard.

-2003. Rodrigue et Chimène, édition de Richard Langham Smith. Paris : Durand.

Ferris, David. 200o. Schumann's Eichendorff Liederkreis and the Genre of the Romantic Cycle. New York : Oxford University Press.

Genette, Gérard. 1987. Seuils. Paris : Seuil. 
Gut, Serge. 1980. "Canope de Debussy, analyse formelle et structure fondamentale ». Revue Musicale de Suisse Romande 33, $\mathrm{n}^{0} 2$ : 60-65.

Hoeckner, Berthold. 1997. «Schumann and Romantic Distance». Journal of the American Musicological Society 50, $\mathrm{n}^{\mathrm{O}} 1:$ :55-132.

Howat, Roy. 1992. "Chopin's Influence on the Fin de Siècle and Beyond ». The Cambridge Companion to Chopin, sous la dir. de Jim Samson, 246-283. Cambridge : Cambridge University Press.

_ 1995. «En route for L'île joyeuse: The Restoration of a Triptych ». Cahiers Debussy $19: 37-52$.

Jankélévitch, Vladimir. 1976. Debussy et le Mystère de l'instant. Paris : Plon. 1949. Debussy et le Mystère. Neuchâtel (Suisse) : Éditions de la Baconnière.

Kallberg, Jeffrey. 1996. Chopin at the Boundaries: Sex, History, and Musical Genre. Cambridge : Harvard University Press.

Kritzman, Lawrence D. et Jeanine Parisier Plottel (dir.). 1981. Fragments: Incompletion and Discontinuity. New York: New York Literary Forum.

Langham Smith, Richard. 1989. "Tonalities of Darkness and Light ». Dans Claude Debussy: Pelléas et Mélisande, sous la dir. de Roger Nichols et Richard Langham Smith, 107-139. Cambridge : Cambridge University Press.

Médicis, François de. 2005. «La polytonalité selon Darius Milhaud : 'Plus subtile dans la douceur, plus violente dans la force...' ». Polytonalité/Polymodalité, sous la dir. de Michel Fischer et Danièle Pistone, 91-115. Paris : Université de Paris-Sorbonne, Observatoire musical français.

- 2007. «Tristan dans La Mer: Le crépuscule wagnérien noyé dans le zénith debussyste? ». Acta musicologica 79, $\mathrm{n}^{0} 1$ : 195-251.

Meyer, Leonard B. 1991. " A Pride of Prejudices; Or, Delight in Diversity. » Music Theory Spectrum 13, $\mathrm{n}^{\mathrm{O}} 2:$ 241-251.

Rosen, Charles. 1995. The Romantic Generation. Cambridge : Harvard University Press.

Satyendra, Ramon. 1997. "Liszt's Open Structures and the Romantic Fragment ». Music Theory Spectrum 19, $\mathrm{n}^{\mathrm{O}} 2: 184^{-205}$.

Warburton, Thomas. 1982. "Bitonal miniatures by Debussy from 1913 ». Cahiers Debussy-Nouvelle série $6:$ 5-15.

Watkins, Glenn. 1994. Pyramids at the Louvre: Music, Culture, and Collage from Stravinsky to the Postmodernists. Cambridge : Harvard University Press.

FrançOIS DE MÉdicis 